\title{
Estrés hídrico en México bajo dos enfoques de huella de agua de escasez
}

\section{Water stress in Mexico under two water footprint approaches}

\author{
Heryka Annette Olier Sarlat ${ }^{1}$ \\ Instituto Interamericano de Tecnología y Ciencias del Agua (IITCA) (México)
}

Recibido: 24 de septiembre de 2018

Aceptado: 01 de enero de 2019

Publicado: 19 de junio de 2019

\section{Resumen}

La escasez de agua tiene lugar cuando la demanda supera el suministro de agua dulce en un área determinada (FAO, 2013). La huella de agua es una herramienta que pretende evaluar los impactos potenciales relacionados con el uso del agua, aportando información con respecto a la escasez, disponibilidad, ecotoxicidad, eutrofización y acidificación. El concepto de huella implica la medición de la cantidad total de impactos al ambiente producidos directa o indirectamente por productos o procesos en todas las etapas de su ciclo de vida.

Cuando la huella de agua es orientada al estrés hídrico, es posible integrar al volumen total del líquido utilizado, los impactos ambientales provocados en la elaboración de un producto o proceso y, de esta manera,

\footnotetext{
${ }^{1}$ Email: maulei@hotmail.com
} 
proporcionar información para el establecimiento de medidas para mitigar o eliminar la huella, tendiendo a la optimización en la utilización de los recursos hídricos.

En esta investigación se revisan los criterios y procedimientos de los indicadores AWARE (Boulay et al., 2017) y WSI (Pfister et al., 2009) para la evaluación de huella de escasez de agua, considerando datos nacionales de las regiones hidrológicas que conforman el territorio mexicano en una escala anual.

El análisis comparativo de los indicadores AWARE y WSI, han sido el primer paso para la evaluación de los impactos provocados por el uso de agua en los recursos hídricos en México. Los dos indicadores fueron evaluados a nivel regional con datos nacionales, teniendo coincidencias en las zonas con mayor estrés hídrico, pero con limitaciones sobre la concepción de las fuentes y definición de uso. La comprensión de los conceptos utilizados en ambos indicadores, son adaptados a los establecidos en México, como variables que pueden influir en los resultados de ambos indicadores.

Palabras clave: AWARE, WSI, escasez, huella de agua. 


\section{Abstract}

Water scarcity occurs when demand exceeds the supply of fresh water in a given area (FAO, 2013). The water footprint is a tool that aims to assess the potential impacts related to water use, providing information regarding scarcity, availability, ecotoxicity, eutrophication and acidification. The concept of footprint involves the measurement of the total amount of environmental impacts produced directly or indirectly by products or processes at all stages of their life cycle.

When the water footprint oriented to the water stress index, it is possible to integrate the total volume of the liquid used, the environmental impacts caused in the elaboration of a product or a process and, in this way, provide information for the establishment of measures to mitigate or eliminate the footprint, tending to optimize the use of water resources.

This research reviews the criteria and procedures of the AWARE (Boulay et al., 2017) and WSI (Pfister et al., 2009) indicators for the assessment of scarcity water footprint, considering national data of the hydrological regions that make up the Mexican territory on an annual scale.

The comparative analysis of the AWARE and WSI indicators, has been the first step for the evaluation of the impacts caused by the use of water in the water resources in Mexico. The two indicators were evaluated at the regional level with national data, showing coincidences in the areas with the greatest water stress, but with limitations on the conception of the sources and definition of use. The understanding of the concepts used in both indicators is adapted to those established in Mexico, as variables that can influence the results of both indicators.

Keywords: AWARE, WSI, scarcity, water footprint. 


\section{Introducción}

La huella de agua es la medición de la cantidad total de impactos ambientales relacionados con el agua usada en un producto, proceso o servicio (Kounina et al., 2012). Los métodos para evaluar los impactos asociados con el uso del agua se encuentran estandarizados en la norma ISO 14046:2014 Gestión ambiental-Huella de agua (principios, requisitos y directrices). La evaluación de los impactos se realiza con base en la técnica de análisis de ciclo de vida ACV (NTC-ISO 14040-2007), donde es posible considerar indicadores de escasez para la evaluación de los impactos potenciales por consumo de agua dulce. En términos del ISO esta evaluación es connotada como un reporte no integral asociado a impactos de punto medio sobre cantidad de agua usada (Boulay et al., 2017). El reporte integral de la huella de agua es aquel que evalúa todas las categorías de impacto tanto en punto intermedio (impacto) como en punto final (daño).

El reporte no integral de la huella de agua, contempla sólo una categoría de impacto, agregando al reporte el nombre del calificador de impacto (p.e. huella de escasez de agua).

Estas categorías proporcionan el impacto en el uso de agua utilizada en metros cúbicos de agua equivalentes $\left(\mathrm{m}^{3} \mathrm{eq}\right)$. Esta conversión es realizada por medio de coeficientes llamados factores de caracterización, derivados de diversos métodos.

Un factor de caracterización surge de un modelo analítico que contiene el análisis de una base de datos relacionada con un indicador de categoría (escasez, disponibilidad, eutrofización, ecotoxicidad, etc.).

A partir de la publicación de la norma ISO 14046 (2014), se han realizado algunos estudios de huella de agua aplicando factores de caracterización para las diferentes categorías. Su aplicación ha sido observada en procesos tales como la fabricación de cemento, o de cerveza, así como productos agrícolas (Farell et al., 2013; Álvarez et al., 2013; Vallejo, 2015). De esta manera, existen factores de caracterización para evaluar la huella de agua de uso público y los ecocostos de la huella de agua como forma de reportar los impactos ambientales en términos monetarios (Embarcadero, 2014; Farell et al., 2013).

Organizaciones como UNEP (United Nation Enviroment Programme)-SETAC (Society of Environment Toxicology and Chemistry) (2012) e ISO (International Organization for Standardization) (2017), desarrollan indicadores por categorías de impacto. Uno de estos impactos es la vulnerabilidad sobre los recursos hídricos ya sea en términos de escasez o estrés hídrico (WULCA, 2014, Brown \& Matlock, 2011). Esta investigación revisa los criterios y procedimientos de los indicadores AWARE (Boulay, 2011) y WSI (Pfister et al., 2009) para la evaluación del estrés hídrico bajo la categoría de huella de escasez de agua, 
considerando datos nacionales de las regiones hidrológicas que conforman el territorio mexicano en una escala anual.

\section{Materiales y Métodos}

Tanto el indicador WSI, como el AWARE utilizan información común referente al uso y la disponibilidad del agua. Por ello, la presente investigación considera datos provenientes de fuentes oficiales. Sin embargo, posteriormente a la estimación de los indicadores, se abre una discusión sobre las limitaciones encontradas en los resultados tales como las diferencias conceptuales en los términos de uso, así como las implicaciones de la estimación de la disponibilidad en aguas superficiales y subterráneas.

\subsection{Indicador de Estrés Hídrico WSI}

El indicador Water Stress Index, (WSI), asociado a un impacto por privación de agua (Pfister et al., 2009), indica la relación entre consumo y disponibilidad de agua, en una cuenca específica. Matemáticamente, el WSI relaciona el agua para consumo humano y la disponibilidad natural de ésta, calibrado con datos hidrológicos globales para obtener valores continuos entre 0 a 1 (Ec. 1).

$$
W S I=\frac{1}{1+e^{-6.4 * W T A^{*}}\left(\frac{1}{0.01}-1\right)}
$$

El indicador considera los sistemas hidrológicos y la parte socioeconómica, para cuantificar la disponibilidad anual de agua dulce $\left(W A_{i}\right)$, y la extracción por parte de diferentes usuarios $j\left(W U_{i j}\right)$, para cada cuenca $i$. Aunque se ha aplicado principalmente en regiones áridas a nivel mundial (Boulay, et al., 2017), el impacto en los recursos hídricos es mayor. Se sugiere un enfoque de modelado regionalizado para la evaluación de consumo de agua. Esta observación es relevante para la toma de decisiones enfocadas a la cadena de suministro de un producto o bien para los consumidores interesados en un consumo sostenible (Farell, 2013). El grado de estrés hídrico WTA* (Withdrawal To Availability; Ec. 2; Nilsson et al., 2005) de una cuenca i , depende del volumen de agua disponible anualmente $\left(W A_{i}\right)$ y del volumen de aguaextraída para los usuarios $\left(W U_{i j}\right)$ en donde los resultados se expresan en $m^{3}$ equivalente. 


$$
\begin{gathered}
W T A^{*}=\sqrt{V F} * \sum_{j} \frac{W U_{i j}}{W A_{i}} \quad \text { para SRF } \\
\mathrm{VF}^{*} \sum_{j} \frac{W U_{i j}}{W A_{i}} \quad \text { para No SRF }
\end{gathered}
$$

Donde SRF son caudales fuertemente regulados y VF=1.8 (Pfister, 2012) es un factor de variación (Ec. 3), que se basa en la desviación estándar tanto mensual $\left(S_{m e s}^{*}\right)$ como anual $\left(S_{a n ̃ o}^{*}\right)$ de la precipitación, asumiendo una distribución log-normal (Pfister et al., 2009).

$$
V F=e^{\sqrt{\left[\ln \left(S_{m e s}^{*}\right)\right]^{2}+\left[\ln \left(S_{a \tilde{n} o}^{*}\right)\right]^{2}}}
$$

Un WSI con valores menores a 0.09 indica un estrés hídrico bajo (WTA*<0.2); un WSI de 0.09 a 0.5 indica un estrés hídrico medio (WTA*<0.4); un WSI de 0.5 a 0.91 un estrés alto (WTA*<0.6) y un WSI de 0.91 a 1 representa un estrés hídrico muy alto

El término de disponibilidad en el método WSI se basa en el correspondiente al modelo WaterGAP (Alcamo et al., 1997), el cual estima flujos como escurrimiento superficial asociados a una cuenca hidrográfica a partir de la variación anual de precipitación. El estrés hídrico puede ser incrementado durante períodos específicos si el almacenamiento de agua es insuficiente, reduciendo la capacidad de disponibilidad

\subsection{Remante de Agua Disponible AWARE}

Uno de los métodos para estimar el estrés hídrico como la diferencia entre el uso de agua total y la disponibilidad de agua es el indicador AWARE (Available Water Remaining). Es un indicador regionalizado que representa el remanente de agua disponible por área en una cuenca hidrográfica después de que se haya satisfecho la demanda de los seres humanos y los ecosistemas (Boulay et al., 2017). Se basa en la suposición de cuanto menor sea el agua disponible por área, más probable es que otro usuario será privado de este recurso. AWARE es el método recomendado por el grupo Water Use in Life Cicle Assessment (WULCA, 2017), El factor de caracterización de AWARE (FCAWARE; Ec. 4), es un valor con rango entre 0.1 y 100 y está en función de la diferencia entre disponibilidad y demanda AMD (Availability Minus Demand) por unidad de superficie relativo a un promedio mundial equivalente $A M D_{\text {prommund }}$ igual con $0.0136 \mathrm{~m}^{3} / \mathrm{m}^{2 *} \mathrm{mes}$. Un 
$F C_{A W A R E}$ igual con 1 corresponde a una región con la misma cantidad de agua por área dentro de un cierto período de tiempo igual al promedio mundial.

Valores menores a 1 representan regiones, con poco estrés hídrico en relación con el promedio mundial. Una región con $F C_{A W A R E}$ de 10, representa un requerimiento 10 veces más grande de dicha área para generar la cantidad promedio mundial de agua. El valor máximo de 100, representa regiones donde la demanda es mayor que la disponibilidad.

$$
F C_{A W A R E}=\left\{\begin{array}{cc}
\frac{A M D_{\text {prom mund }}}{A M D} & \text { si demanda }<\text { disponibilidad } \\
100 & \text { en otro caso }
\end{array}\right.
$$

El término AMD (Ec. 5) es la diferencia de la disponibilidad (availability) y las aguas consumidas por el hombre (HWU) y las aguas requeridas para el medio ambiente (EWR) relativas a una superficie (Área).

$$
A M D=\frac{\text { Availability }-H W U-E W R}{\text { Area }}
$$

Las regiones con AMD 100, son 100 veces menor que el promedio mundial lo que representan el 5\% del consumo mundial del agua (Boulay et al., 2017).

\subsection{Zona de estudio}

Una región hidrológica administrativa (RHA), es un órgano colegiado que integra la planeación, realización y administración de las acciones formadas por agrupaciones de cuencas, consideradas como las unidades básicas para la gestión de recursos hídricos en México (CONAGUA, 2015).

En México existen 722 cuencas de aguas superficiales y 653 acuíferos agrupadas en 37 regiones hidrológicas y 13 RHA (Figura 1).

Para el caso de México la disponibilidad de aguas subterráneas es la relación de la carga y descarga de los diferentes acuíferos (CONAGUA, 2015). Para las aguas superficiales la disponibilidad es el volumen 
de aguas precipitadas menos el agua evaporada, las aguas captadas y distribuidas para diversos usos, adicional a la extracción ya concesionada y a la descarga natural comprometida, sin poner en peligro el equilibrio de los ecosistemas. (LAN, 2016; CONAGUA, 2016).

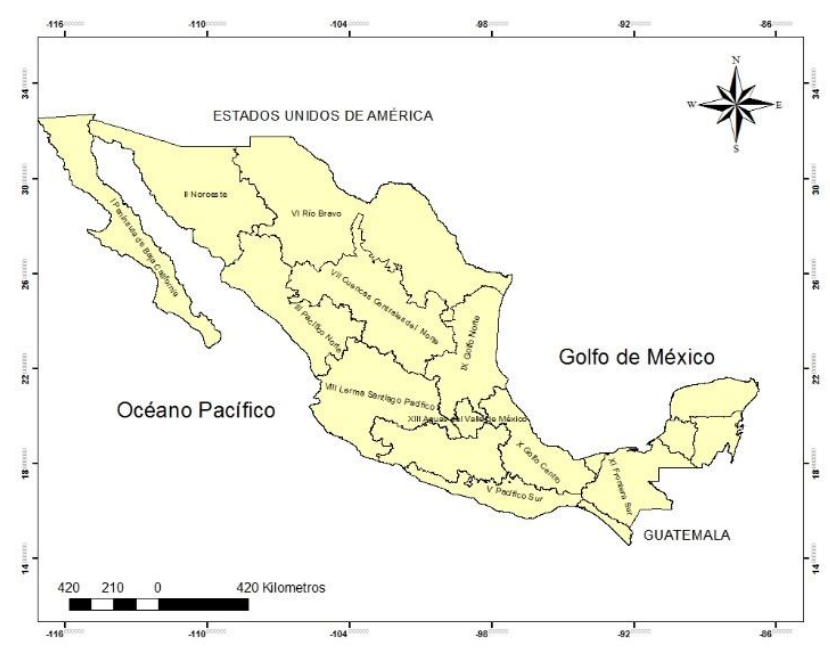

Figura 1. Regiones hidrológicas administrativas en México (CONABIO, 2018).

En relación con el uso del agua, México cuenta con un registro de concesiones de agua REPDA (2015) clasificadas en 12 categorías, las cuales a su vez están agrupadas en cinco categorías. Cuatro de ellas corresponden a uso consuntivo: agrícola, abastecimiento público, industria autoabastecida y termoeléctricas. El quinto grupo son las hidroeléctricas por ser de uso no consuntivo (SINA, 2017). A nivel nacional, hay una disponibilidad de $446778 \mathrm{hm}^{3} /{ }_{a \tilde{n} o}$ y un uso total de $3692 \mathrm{~m}^{3} /$ año por habitante. (CONAGUA, 2015).

\section{Resultados y discusiones}

Los conceptos que conforman el indicador de escasez, principalmente el término de 'disponibilidad hidrológica', fueron comparados con definiciones dadas por en CONAGUA (2015), SEMARNAT (2010), ISO (2014), la Ley de Aguas Nacionales y de los autores de los métodos AWARE y WSI. De esta manera, fue asociado el término volumen usado con volumen concesionado en función del acceso a las fuentes de información. Adaptar el concepto de agua usada para los términos utilizados en México, implica un desafío ya que aguas concesionadas (REPDA, 2018), se refiere a las aguas que son asignadas por una unidad federativa. Sin embargo, este concepto difiere en cierto grado con los términos volúmenes demandados y extraídos, las 
aguas demandas son aquellas que el usuario requiere para satisfacer sus necesidades y las aguas extraídas es aquel volumen de agua que ha sido medido en campo.

Por otro lado, CONAGUA (2015) el agua máxima factible de explotar anualmente en una región, la cual es renovada por la lluvia y el agua proveniente de otras regiones o países (Tabla 1).

Las aguas renovables están determinadas por la diferencia de los flujos de entrada y salida de un sistema. Para obtener los volúmenes de aguas renovables, se debe estimar los flujos de entrada, determinados como la suma de la precipitación anual, las importaciones de agua, el escurrimiento natural medio superficial, la recarga total anual de los acuíferos y los flujos de entrada menos los flujos de salida de agua a otras regiones (Gleick, 1990). Los flujos de salida están conformados por la suma de la evapotranspiración y la exportación de agua.

Tabla 1.

Datos oficiales para las variables de disponibilidad y de uso de agua para las trece regiones hidrológicas administrativas en México

\begin{tabular}{cccc}
\hline RHA & $\begin{array}{c}\text { A. } \\
\text { renovables } \\
\left(\frac{\boldsymbol{h m}^{\mathbf{3}}}{\boldsymbol{a n ̃ o}}\right)\end{array}$ & $\begin{array}{c}\text { A. } \\
\text { Concesionadas } \\
\left(\boldsymbol{h m}^{\mathbf{3}} / \boldsymbol{a} \tilde{\text { no }}\right)\end{array}$ & $\begin{array}{c}\text { Población } \\
(\text { millones } \\
\text { de hab. })\end{array}$ \\
\hline XIII & 3458 & 4771 & 23.01 \\
I & 4958 & 3949 & 4.37 \\
VI & 12316 & 9513 & 12.15 \\
II & 8273 & 6715 & 2.80 \\
VII & 7849 & 3801 & 4.52 \\
IV & 22156 & 10784 & 11.69 \\
III & 25596 & 10731 & 4.47 \\
VIII & 35093 & 15292 & 23.89 \\
IX & 28085 & 5899 & 5.23 \\
XII & 29324 & 4149 & 4.52 \\
V & 30565 & 1539 & 5.02 \\
X & 95129 & 5449 & 10.48 \\
XI & 144459 & 2337 & 7.57 \\
\hline
\end{tabular}


En relación con el WSI (Tabla 2) las zonas con mayor estrés hídrico se presentan en el centro y norte del territorio nacional (Figura 2). Siendo las aguas del Valle de México la región con estrés severo (WSI=0.99) y las regiones del sur con los valores de estrés más bajos.

Tabla 2.

Aplicación de los métodos WSI y AWARE para las trece regiones hidrológicas administrativas en México

\begin{tabular}{lll}
\hline \multicolumn{1}{c}{ RHA } & WSI & AWARE \\
\hline XIII Aguas del Valle de México & 0.99 & 100 \\
I Península de Baja California & 0.98 & 25.31 \\
VI Río Bravo & 0.98 & 22.63 \\
II Noroeste & 0.99 & 20.69 \\
VII Cuencas Centrales del Norte & 0.73 & 7.49 \\
IV Balsas & 0.73 & 1.66 \\
III Pacífico Norte & 0.56 & 1.65 \\
VIII Lerma-Santiago-Pacífico & 0.60 & 1.57 \\
IX Golfo Norte & 0.10 & 0.93 \\
XII Península de Yucatán & 0.05 & 0.91 \\
V Pacífico Sur & 0.01 & 0.46 \\
X Golfo Centro & 0.019 & 0.19 \\
XI Frontera Sur & 0.01 & 0.11
\end{tabular}




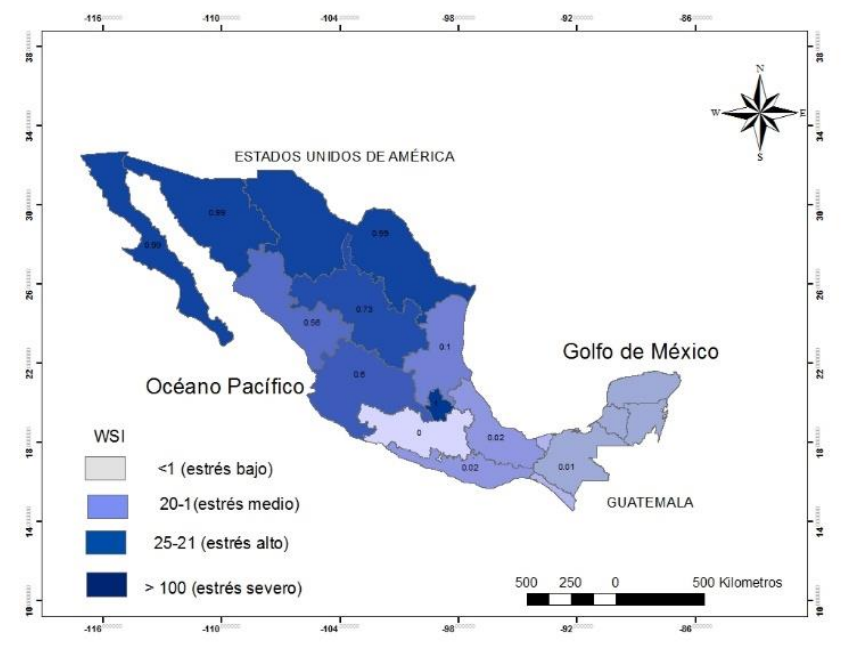

Figura 2. Estrés hídrico (WSI) para las trece regiones hidrológicas administrativas en México, (Elaboración propia, 2018).

Los resultados obtenidos en cada RHA (Figura 3), demostró la desigual distribución de los recursos hídricos en México, además de su desigual uso (Ejemplo de esto se ven las RHA VI, I, II que son las que se encuentran al norte del territorio nacional y que presentan niveles de precipitación inferiores a las RHA XII, VXI siendo estas las regiones con mayores niveles de precipitación).

El FC $\mathrm{AWARE}_{\mathrm{A}}$ en las trece RHA (Figura 3) muestra, al igual que el WSI, que las zonas con mayor estrés hídrico se presentan en el centro y norte del territorio nacional. Ambos indicadores muestran una coherente clasificación de los recursos hídricos por estrés hídrico. Sin embargo, resalta la dispersión entre las variaciones de sus valores. Por ejemplo, la variación del $\mathrm{FC}_{\mathrm{AWARE}}$ entre la primera y segunda región con mayor estrés hídrico es del $75 \%$, mientras que en el WSI es apenas del $1 \%$.

Los resultados que se obtuvieron aplicando el AWARE para las diferentes RHA en México, mostraron entre las regiones con mayor impacto, a la RHA XIII (Aguas del Valle de México) con un Factor AWARE de 100, siendo está región la más poblada del país. La región I con un AWARE de 25.31, corresponde a la Península de Baja California que, aunque no es la más poblada ni con mayor extensión de superficie, es la segunda región que presenta un mayor impacto potencial en los recursos hídricos, pudiendo influenciar las condiciones de sequía que prevalecen en esta región. 


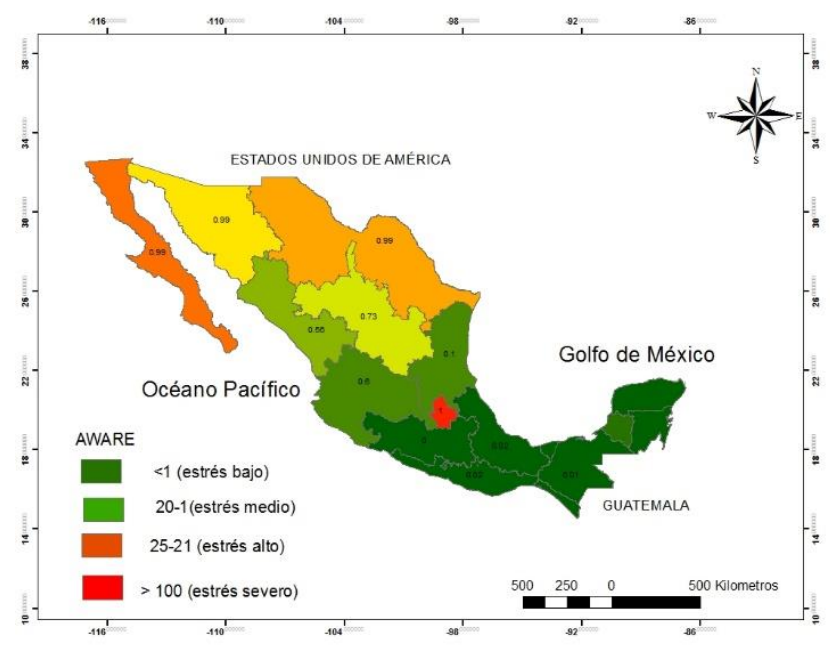

Figura 3. Remanente de agua disponible (AWARE) para las trece regiones hidrológicas administrativas en México (Elaboración propia, 2018).

La clasificación de las RHA con el WSI muestra una distribución menos dispersa. Los valores más altos se presentaron en las regiones con mayor población, y con condiciones de sequía extremas como son las regiones I y VI que presentan condiciones de sequía con intensidades de DO (anormalmente seca) (CONAGUA, 2014), regiones XIII Aguas del Valle de México con un impacto potencial de 0.99, VI Río Bravo con un WSI de 0.98 y la región I Península de Baja California con 0.98.

A pesar de la coherencia en el orden de impacto por RHA, la clasificación observada en la tabla 1, muestra ciertas variaciones. En algunos casos, como en la RHA XIII el orden de clasificación fue afectado por la extensión territorial.

De manera adicional, ambos indicadores dependen de un balance hídrico general. Lo anterior implica que cuerpos de agua sobreexplotados no son reflejados de manera adecuada en estos indicadores. Por ejemplo, la RHA VIII muestra valores de WSI $=0.6$ y FC $\mathrm{AWARE}=1.57$ mientras que cuenta con 32 de 128 acuíferos sobreexplotados y asociados a zonas metropolitanas principalmente (CONAGUA, 2017).

Cabe resaltar que, aunque en la estimación de ambos indicadores se utilizaron datos de concesión, en otros estudios (Boulay, 2011; Bayart et al., 2010; Pfiter et al., 2012) se han considerado datos de demanda o extracción. Lo anterior proporciona la posibilidad de extender el término de uso a tres connotaciones diferentes. 
De manera adicional, es importante resaltar que en la diferencia entre las estimaciones de aguas renovables y el agua usada no es considerado el potencial volumen de retorno derivado de las actividades agrícolas, fugas en las redes de abastecimiento urbano, descargas industriales, entre otras (CONAGUA, 2015).

\section{Conclusiones}

El análisis comparativo de los indicadores AWARE y WSI, ha sido el primer paso para una evaluación de los impactos provocados por el uso de agua en los recursos hídricos en México. Los resultados de la evaluación del estrés hídrico, permiten determinar los riesgos ambientales provocados por las diferentes actividades que cada región presenta. Ambos métodos presentaron un FC regionalizados coincidiendo con las regiones con mayor escasez de agua. El método WSI demostró mayor sensibilidad a la escasez local.

Una de las limitantes al replicar estos indicadores fue la homogenización de los conceptos con base en los propuestos por los autores. El desarrollo de la base de datos contiene datos limitados sobre el uso del agua y los diferentes conceptos que encierran el término de disponibilidad.

Tomando en cuenta que algunas regiones donde son reportadas regiones sobreexplotadas y al aplicar la evaluación de los dos indicadores no se reflejaron las condiciones reales de dichos cuerpos de agua, se sugiere aplicar los indicadores por fuentes separadas.

El cálculo de los indicadores aquí evaluados permite comprender los desafíos del agua y sus riesgos que la humanidad enfrenta, sirviendo este análisis como una primera fase para la toma de decisiones estratégicas para la evaluación de los recursos hídricos en un país que presenta grandes retos para la conservación de sus recursos naturales. 


\section{Referencias}

Alcamo J.,Doll, P., Kaspar, F., Siebert (1997). Global change and global scenarios of water use and availability: An application of WaterGap 1.0. Center for Environmental Systems Research (CESR), University of Kasse, Hermany.

Álvarez, H.J., Larripa, J.M; Galli, J.R.; Civit, B.M. (2013) Inventario de la huella de agua en sistemas lecheros diferenciados por el uso de la tierra y el nivel de suplementación. Facultad de Ciencias Agrarias. CONICET, Mendoza. $36^{\circ}$ Congreso Argentino de Producción Animal.

F. y Koehler, A. (2010) A framework for assessing off-stream freshwater use in LCA.. Int J Life Cycle Assess (2010) 15:439-453pp. DOI: 10.1007/s11367-010-0172-7

Boulay, A.M., Bulle, C., Bayart J.B, Deschênest, Margnit, M. (2011) Regional characterization of freshwater use in LCA: Modeling Direct impacto n human health, Environ. Sci. Technol., 45 (20), pp 8948-8957. DOI: $10.1021 /$ es1030883

Boulay, A.M., Bare, J., Benini,L., Berger, M., Lathulliere, M.J., Manzardo.,Margni,M., Motochita, M., Núñez, M., Pastor, A.V.,Ridoutt,B., Oki, T., Worbe, S., Pfister, S. (2017) The WULCA consensus characterization model for water scarcity footprint: assessing impact of water consumption based on available water remaining (AWARE). Recuperado de: https://www.youtube.com/watch?v=w_gKfV-TgzI

CONAGUA (2014) Atlas del Agua en México 2014. Biblioteca Mexicana del conocimiento.

CONAGUA (2015) Estadísticas del Agua en México, edición 2015. Autor: Comisión Nacional del Agua.

Editor: Secretaría del Medio Ambiente y Recursos Naturales. www.conagua.gob.mx.

CONAGUA (2017) Estadísticas del agua en México, Edición 2017, SEMARNAT, México, 291 p.

CONABIO (2018) Comisión Nacional para el Conocimiento y Uso de la Biodiversidad. http://www.conabio.gob.mx/

Farell, C. (2013) Diseño de una metodología para reportar la huella de agua. Tesis Doctoral. Universidad Autónoma. Metropolitana, Azcapotzalco, México.

Farell, C., Turpin, S., Suppen, N. (2013) Huella de agua de uso público-urbano en México. Realidad, datos y espacio. Revista Internacional de estadística y geografía. (1) 58-71. 
Gleick P.H. (1990) Global climatic change and international security, Colorado Journal of International Environmental Law and Policy, 1 (1), pp. 41-56.

ISO 14044 (2006) Environmental Management-Life Cycle Assessment-Principles and Framework (ISO 14044:2006); European Committee for Standardisation: Brussels, Belgium, 2006.

ISO 14046 (2014) International Standard under development. Life Cycle assessment-Water footprintRequirements and guidelines.

Nilsson, C.; Reidy, C. A.; Dynesius, M.; Revenga, C. (2005) Fragmentation and flow regulation of the world's large river systems. Science2005, 308 (5720), 405-408.

NTCISO 14040 (2007) Norma Técnica Colombiana. Gestión ambiental análisis de ciclo de vida. Principios y marco de referencia. 2007

Ley de Aguas Nacionales (LAN) (2016) Diario Ofcial de la Federación. Recuperado de: http://www.diputados.gob.mx/LeyesBiblio/pdf/16_240316.pdf

Pfister, S.,Ridoutt,B., van Zelm, R., Verones, F., Humbert, S. (2012) Review of methods addressing freswater use in life cycle inventory and impact assessment. Int J Life Cycle Assess. DOI : $\underline{10.1007 / \mathrm{s} 11367-012-0519-3}$

REPDA (2017) Registros Públicos de Derechos del Agua. Comisión Nacional del Agua. https://www.gob.mx/conagua/acciones-y-programas/consulta-la-base-de-datos-del-repda

UNEP-SETAC (2012) Fase II (2007-2012): promoviendo el enfoque del ciclo de vida mundialmente e involucrando a la comunidad. United Nations Environment Programme (UNEP) and the Society of Environmental Toxicology and Chemistry (SETAC).

Vallejo, A.L. (2015) Metodología práctica para la cuantificación de la huella de agua en plantas empaquetadoras de banano en Costa Rica. Tesis Doctoral.

SINA (2018) Sistema Nacional de Información del Agua. CONAGUA, Recuperado de $\underline{\text { http://sina.conagua.gob.mx/sina/ }}$

WULCA (2017) Factor AWARE. Recuperado de http://www.wulca-waterlca.org/aware.html 
Olier Sarlat, $\mathrm{H}$.

ISSN 1405-6690 impreso 